\title{
Image Case: Irregular Stricture of The Lower Esophagus: Differential Diagnosis
}

\author{
Ahmed Farouq \\ Tropical Medicine Department, Faculty of Medicine, Zagazig University, Egypt
}

A 56 years old male patient presented by progressive dysphagia to solids and fluids. Barium swallow shows long segment lower esophageal irregular stricture (Figure1). The differential diagnosis of this condition includes cancer esophagus, corrosive stricture and esophageal motility disorder. Further investigations are needed to reach the final diagnosis mainly upper GI endoscopy. In case of cancer esophagus the patient is usually of old age, may be young but with history of long standing GERD, usually associated with anorexia, cachexia and weight loss and may also bleeds, endoscopy usually clinch the diagnosis and take biopsy. Corrosive stricture usually follows an acute insult after ingestion of corrosive material. Motility disorder may occur at any age and upper endoscopy as was seen in this case may be irrelevant.

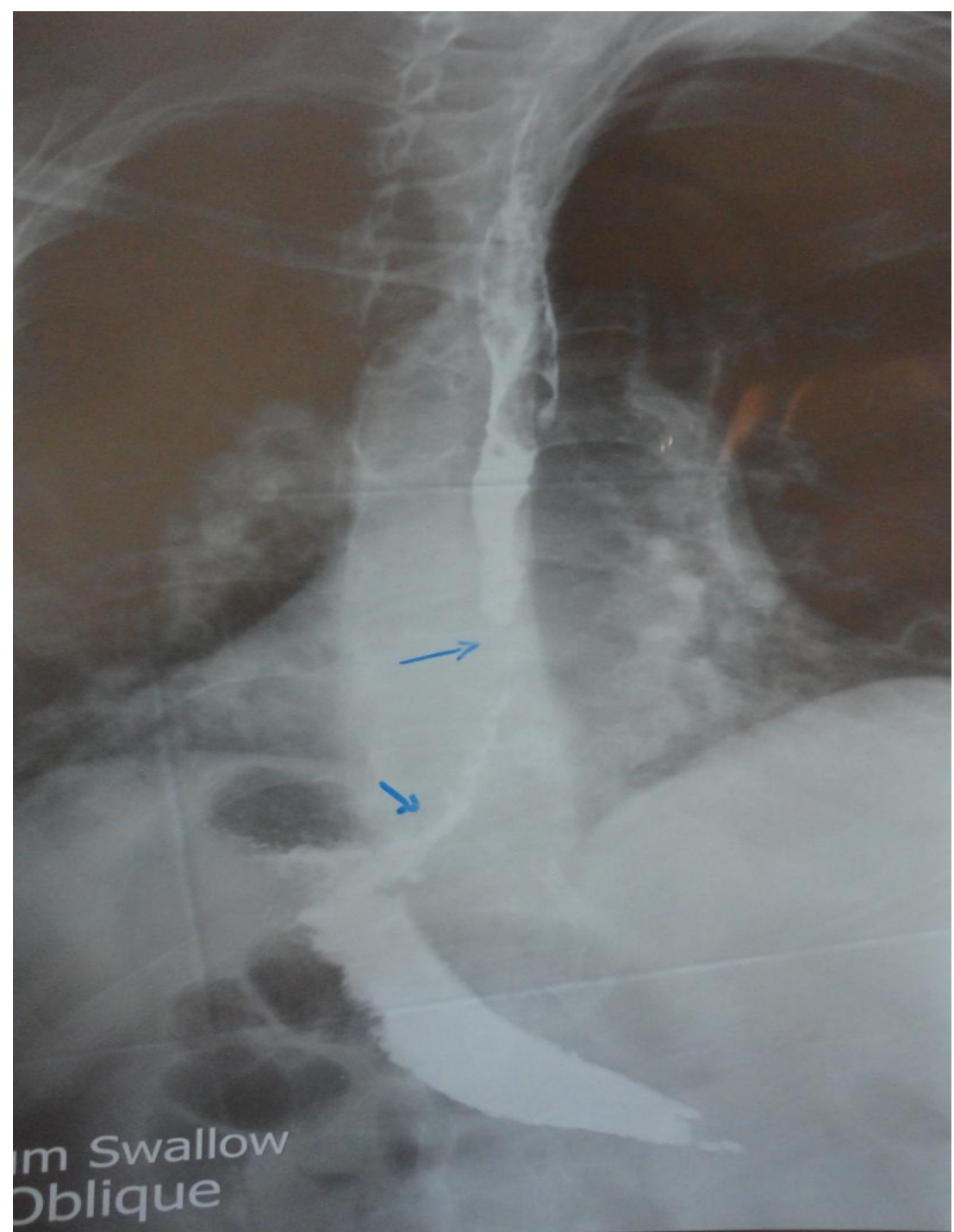

Figure 1: Barium swallow showing long segment lower esophageal irregular stricture 